\title{
Caffeic acid and instant coffee modulate the expression of transcription factors STAT5B and ATF2 in human cancer cells
}

\author{
C. Oleaga ${ }^{1}$, E. Selga ${ }^{1}$, C. Ciudad ${ }^{1}$, M. Izquierdo-Pulido ${ }^{2}$ and V. Noé ${ }^{1}$ \\ ${ }^{1}$ Department of Biochemistry and Molecular Biology and ${ }^{2}$ Department of Nutrition and Food Science, School of Pharmacy, \\ University of Barcelona, Barcelona, Spain
}

Polyphenols represent the major antioxidants intake in our diet and substantial experimental evidence connects polyphenols in the prevention of cardiovascular disease, neurodegenerative disorders, cancer or diabetes. Since ninth century coffee brewed beverage became a social habit, but its high consumption started a hundred years ago. Our aim was to perform nutrigenomic study to identify genes differentially expressed after cell incubation with different coffee polyphenols such as chlorogenic and caffeic acid and other coffee derivatives.

We performed a functional genomic analysis to study the effect of caffeic acid and instant coffee in the human colon adenocarcinoma cell line HT-29. Cells were incubated with caffeic acid or instant coffee at concentrations equivalent to one cup intake. Total RNA was extracted and hybridized to The GeneChip ${ }^{\circledR}$ Human Genome I133A 2.0 microarrays from Affymetrix, containing 54,700 transcripts and variants representing the whole human genome. Results from the microarrays were analysed with the Gene Spring software. The differentially expressed genes, with a statistical significance of $P<0.05$, were classified considering a factor of 1.3 with respect to the expression in the control cells (downregulated: ratio $<0.7$; upregulated: ratio $>1.3$ ). Additionally, we classified the differentially expressed genes according to gene ontology in the following functional groups: cell cycle, transcription factors, immunity, apoptosis and proliferation.

Among the differentially expressed genes, we identified transcription factor STAT5b, which was overexpressed upon cell incubation with either caffeic acid or instant coffee, and transcription factor ATF2, which was underexpressed upon cell incubation with either caffeic acid or instant coffee. Both transcription factors are involved in the response to cytokines and were selected for further validation. Changes in the mRNA levels of STAT5B and ATF2 upon incubation with either caffeic acid or instant coffee were validated by RT-real time PCR and further confirmed at the protein level by Western blot. These results indicated the amount of caffeic acid present in one cup of coffee is able to modulate the expression of genes involved in the immune response in cultured cells. 